PAPER • OPEN ACCESS

\title{
Hardware-software system for magnetic analysis of structure
}

To cite this article: V Kostin et al 2019 J. Phys.: Conf. Ser. 1327012019

View the article online for updates and enhancements.

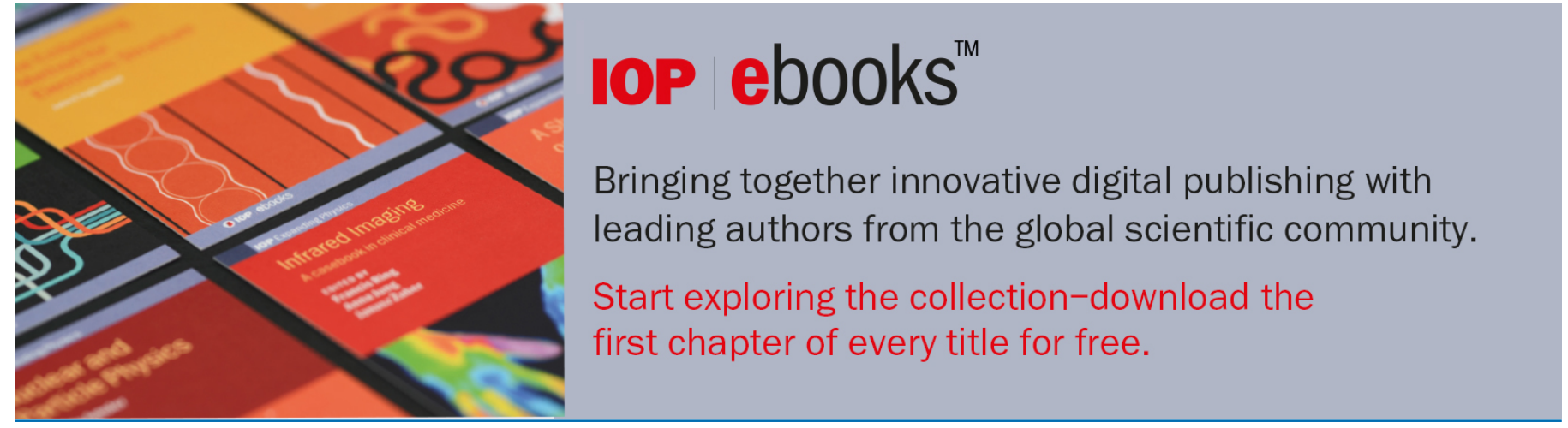

This content was downloaded from IP address 212.193 .78 .232 on $05 / 10 / 2020$ at 12:31 


\title{
Hardware-software system for magnetic analysis of structure
}

\author{
V Kostin ${ }^{1,2}$, O Vasilenko ${ }^{1,2}$ and A Byzov ${ }^{1}$ \\ ${ }^{1}$ M.N. Mikheev Institute of Metal Physics of Ural Branch of Russian Academy of \\ Sciences, Yekaterinburg, Russia \\ ${ }^{2}$ Ural Federal University named after the first President of Russia B.N. Yeltsin, \\ Yekaterinburg, Russia
}

kostin@imp.uran.ru

\begin{abstract}
The relative values of the magnetic properties of a substance is shown to be determined locally by measuring the magnetic field induction in the transducer hole of a special form made in the magnetic circuit of the measuring transducer and the tangential component of the magnetic field in the interpolar transducer space near the surface of the magnetized object.The proposed method is shown to allow for the determination of the whole complex of hysteresis characteristics and significantly expand the ranges of measurement of magnetic properties. The difference in the structural sensitivity of the magnetic properties locally determined in the coordinates "flow - internal field" (properties of matter) and in the coordinates "flow in the product - current in the windings" (properties of the "body") is established.The possibility and expediency of simultaneous measurement and pooled analysis of the magnetic characteristics of the substance and the "body" is shown.Information about the mobile hardwaresoftware system DIUS-1.15M, designed to implement one- and multi-parameter methods of monitoring the structural-phase and stress-strain state of ferromagnetic materials and objects based on the proposed measurement techniques is provided.
\end{abstract}

\section{Introduction}

The development of new methods and devices for the local measurement of magnetic parameters for structuroscopy of ferromagnetic objects is a dynamically developing scientific and technical area. In base, it is concerned with the changes in the structure and phase and also stress and strain state of matter in the FO which static and dynamic magnetic parameters are sensible to [1-4].

For example, the article [5] shows that the measurement of the magnetic field between two permanent magnets, which rotate around a common axis, can be used for investigation of the structure of the ferromagnetic products and determines their stress-strain state. Also, magnetic methods are used to estimate anisotropy [6-8], dislocation density [9], and the effect of tempering temperature on the relaxation coercive force [10]. It is also important to monitor the variation of the magnetic properties of advanced materials in the conditions of changing external conditions, such as temperature [11].

Due to the fact that it is important to use simple, accurate and affordable equipment for physical research, in particular, in magnetic control, the aim of the work was to develop a technique and hardware-software system (HSS) for local measurement of a complex of magnetostatic parameters in a wide range of their changes. 


\section{Methods and materials}

Specially manufactured quality control sample (QCS) with different values of coercive force (CFQCS set) and residual magnetic induction (RMIQCS set) were tested with the aim to prove the elaborated methods and devices for local determination of magnetic values. The CFQCS samples are rectangular parallelepipeds with dimensions of $57 \times 34 \times 7.6 \mathrm{~mm}$, and the RMIQCS set consists of plates with dimensions of $4.6 \times 40.1 \times 90.0 \mathrm{~mm}$.

Variation of properties in a wide range was achieved by the choice of steel grade and subsequent heat treatment (annealing, quenching, tempering). To stabilize the composition and properties, RMIQCS samples with a high content of residual austenite were additionally cold treated at a temperature of $-16^{\circ} \mathrm{C}$ for 3 days.

The magnetic properties of the QCS matter were measured in the permeameter using the measuring equipment REMAGRAPH C-500 manufactured by Magnet-Physik Dr. Steingroever GmbH, Germany (http://www.magnet-physik.de/1.html). The error in measuring magnetization did not exceed $\%$, the field $-1 \%$.

The DIUS-1.15M mobile hardware-software system (HSS) that meets modern requirements $[12,13]$ has been developed for the practical implementation of measurement capabilities. The DIUS-1.15M HSS consists of an attached measuring transducer, measuring block, $24 \mathrm{~V}$ power adapter (or batteries), and a portable computer. A general view of the DIUS-1.15M is shown in Figure 1. The device is operated via a special program that controls the operating modes, visualization and saving the measurement results.

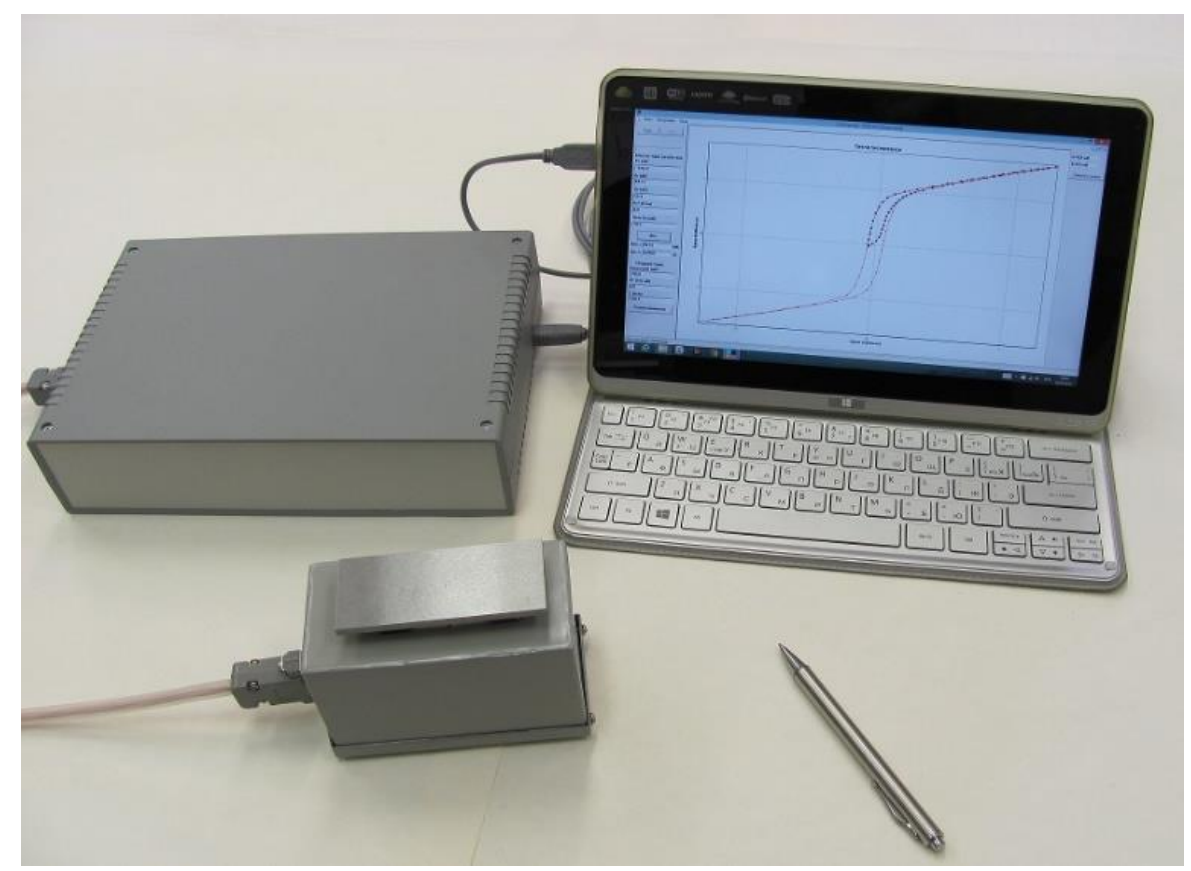

Figure 1. General view of the DIUS-1.15M HSS.

A schematic representation of an attached measuring transducer placed on a tested object (TO) is presented in Figure 2. The converter consists of a continuous U-shaped magnetic circuit (a) with two magnetizing windings (c) and a converter-hole (b).

The transducer-hole is made as a narrow slot with plane-parallel walls perpendicular to the direction of the magnetic flux. The relative magnitude of the magnetic flux in the TO can be determined at the measurement of the magnetic field in the transducer hole (Hall sensor $\mathrm{x} 1$ in Figure 2), and, taking into account the constancy of the cross-sectional area of the magnetized area of the TO, relative magnitude of the induction can be determined in this area. 
It is possible to measure the tangential component of the field on the surface of an object in the interpolar space (Hall sensor $x 2$ in Figure 2) and the magnetic flux simultaneously. Since, as it is known from $[1,15]$, the tangential component does not change at the boundary of two media (according to the boundary conditions), the strength of the magnetic field measured near the surface (the boundary of two media: air and (in case of current work) steel) is almost equal to the internal field in the object. Also, it is necessary to notice, that the proper measurement of the internal field removes limitations on the range of measured magnetic properties.

Thus, it is possible to locally determine its magnetic properties via measuring the relative magnitude of the flux and the internal field in the TO.

\section{Results and discussion}

The described measuring transducer was used both in the DIUS-1.15M and SIMTEST [16]. The magnetic properties of the TO were determined when measuring the relative magnitude of the flow and internal field in the TO on the basis of the flow-internal field hysteresis characteristic by constructing a magnetic hysteresis loop with help of the transducer.

However, the number of parameters can be increased by registering the current in the electromagnet windings. This feature allows us to measure the characteristics of the loop in the coordinates of the "flow in the object - the current in the windings". They could be called the characteristics of the "body", since they depend on the geometrical dimensions of the TO and other parameters of the composite "converterobject" circuit largely.

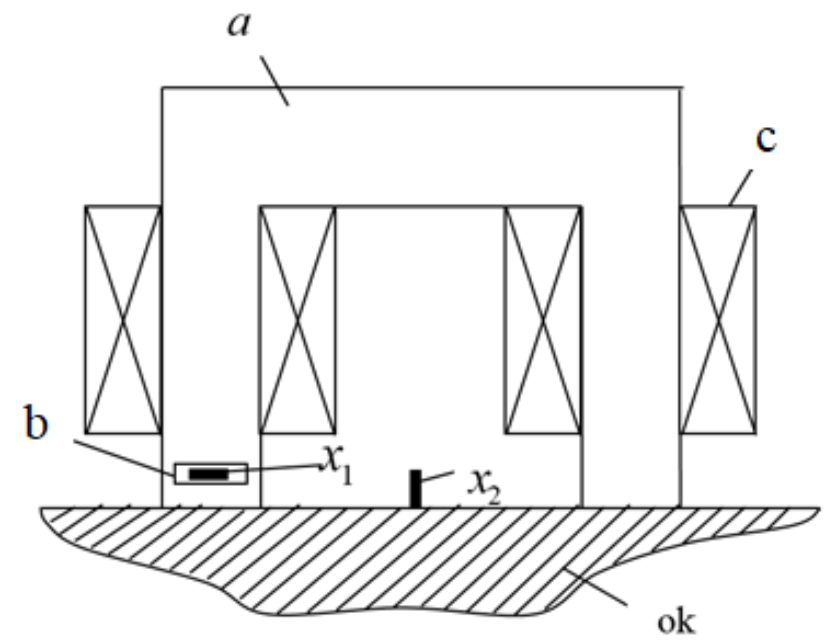

Figure 2. Schematic representation of a side-mounted electromagnet with a hole-converter of the magnetic flux into the magnetic field strength and a gauge of the tangential component of the strength of the internal field: $\mathrm{a}$ - U-shaped magnetic core; $\mathrm{b}$ - hole-converter; $\mathrm{c}$ - magnetizing windings; ok TO; x1, x2 - Hall sensors.

Figure 3 shows the hysteresis loops of the substance and the "body", measured by the converter shown in Figure 2 with a cross section of $12 \times 28 \mathrm{~mm}$ poles for four samples with dimensions $7.5 \times 34 \times 57$ $\mathrm{mm}$, having different values of coercive force.

Also, calibration graphs (see Figure 4) were determined to measure the coercive force in the range $(2.5 \div 47) \mathrm{A} / \mathrm{cm}$ and the residual magnetic induction in the range $(0.2 \div 1.6) \mathrm{T}$ on the sets samples of coercive force and samples of residual magnetic induction by the attached transducer with a cross section of $12 \times 28 \mathrm{~mm}$ poles. Selected ranges cover the largest range of possible applications of the device. However, the capabilities of the DIUS-1.15M HSS are much wider.

The hysteresis loop in the coordinates "flow in the object - current in the windings" can be measured in the objects with any size and shape. In this case, the measurement of the coercive force of the "body" is similar to measurements using such well-known coercimeter as KIFM-1 and it can be used to solve 
the wide range of tasks [1]. However, as can be seen from Figure 5a, the sensitivity to the coercive force of the relative value measured locally according to the internal field in the TO is greater than that of the value determined from the demagnetizing current. Moreover, there is no correlation between the residual magnetic induction and the flow in the object defined in the coordinates "current in the windings" relative value, and the linear correlation coefficient between the quantities and is $\mathrm{R}=0.99$ (Figure 5b). Thus, measurements in the coordinates "flow in the product - internal field" are more preferable.

Nevertheless, the pooled analysis of the properties of matter and the "body" provides much more opportunities for structuroscopy. For example, in $[16,17]$, it is shown that the additional consideration of the characteristics of the "body" can reduce the interfering influence of the uncontrolled gap in the "converter-object" circuit on the results of local measurement of such magnetic parameters of a substance as coercive return induction and coercive force.

Also the calibration characteristics of the same attached transducer were obtained for the ranges of variation of the coercive force $(0.7 \div 6.5) \mathrm{A} / \mathrm{cm}$ and $(15 \div 90) \mathrm{A} / \mathrm{cm}$ (see Figure 6). Based on the constructed dependencies, it can be confirmed that the using such a converter is possible to measure small values of the coercive force, and the minimum value of the measured coercive force equal to 0.7 $\mathrm{A} / \mathrm{sm}$ may be less than the coercive force of the magnetic circuit, which is equal to $1.5 \mathrm{~A} / \mathrm{sm}$. From the data obtained it is clear that the readings of the DIUS-1.15M HSS increase linearly with the growth of the coercive force of the TO up to values of $75 \mathrm{~A} / \mathrm{cm}$. The decrease in the sensitivity of the device at higher values of the coercive force is obviously due to the insufficient magnitude of the magnetomotive force generated by this attached transducer.
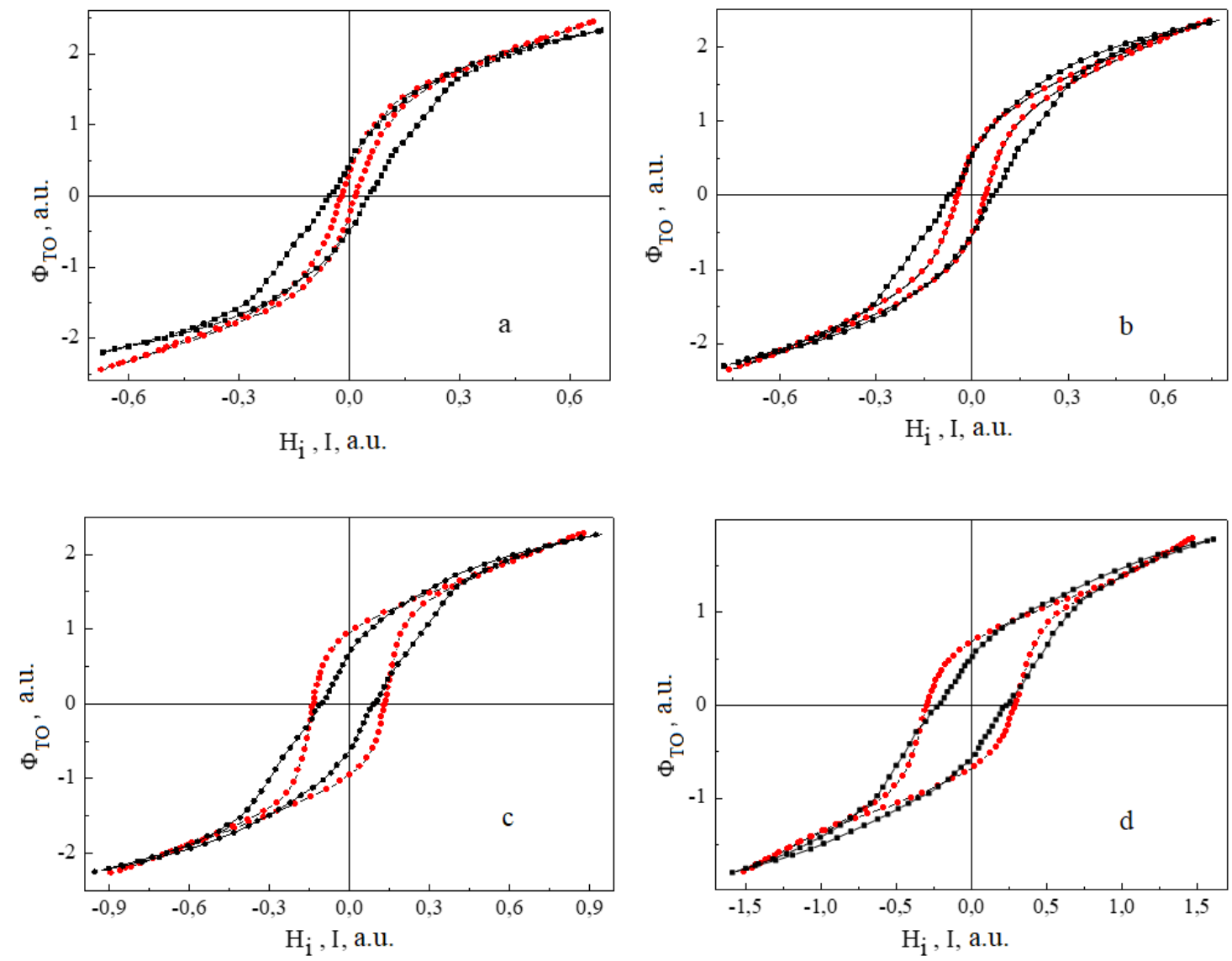

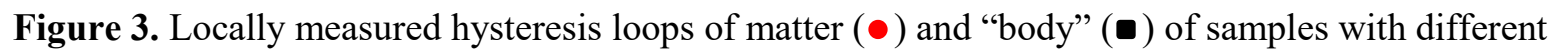
values of coercive force $(\mathrm{Hc})$ : $\mathrm{a}-2.7 \mathrm{~A} / \mathrm{cm} ; \mathrm{b}-6.3 \mathrm{~A} / \mathrm{cm} ; \mathrm{c}-19.8 \mathrm{~A} / \mathrm{cm} ; \mathrm{d}-46.6 \mathrm{~A} / \mathrm{cm}$. 

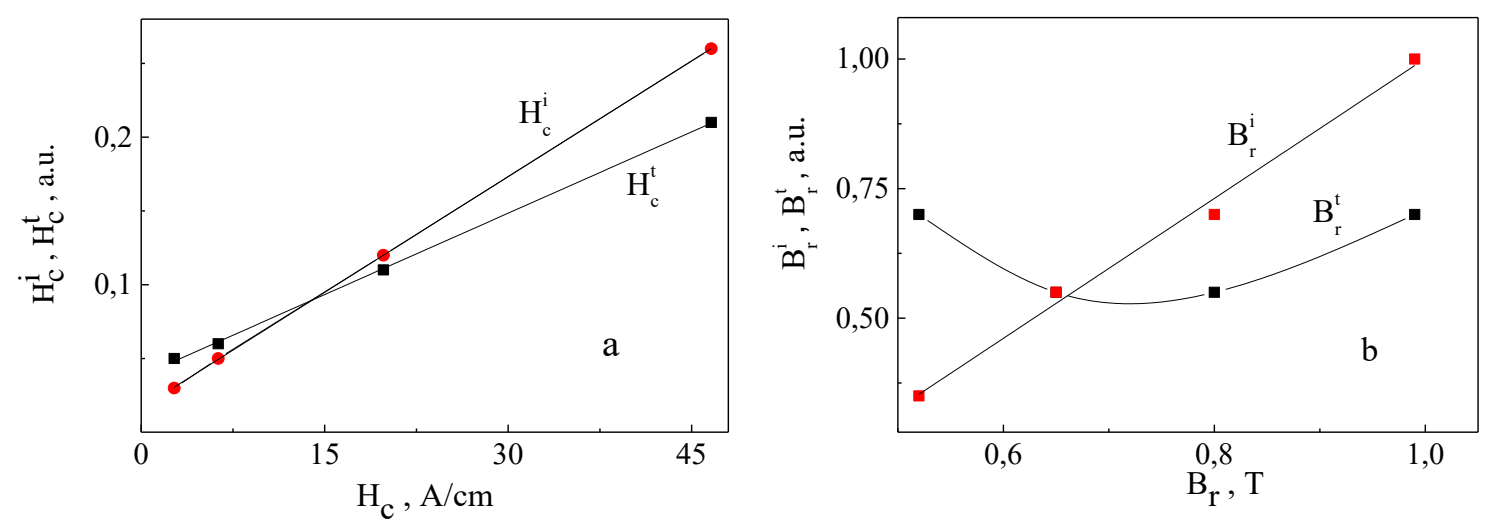

Figure 4. Dependences of locally measured APS DIUS-1.15M values of coercive force (a) and residual magnetic induction (b) of substance ( $\bullet$ ) and "body" ( $\bullet$ ) on measured (true) values of these magnetic properties in the permeameter.
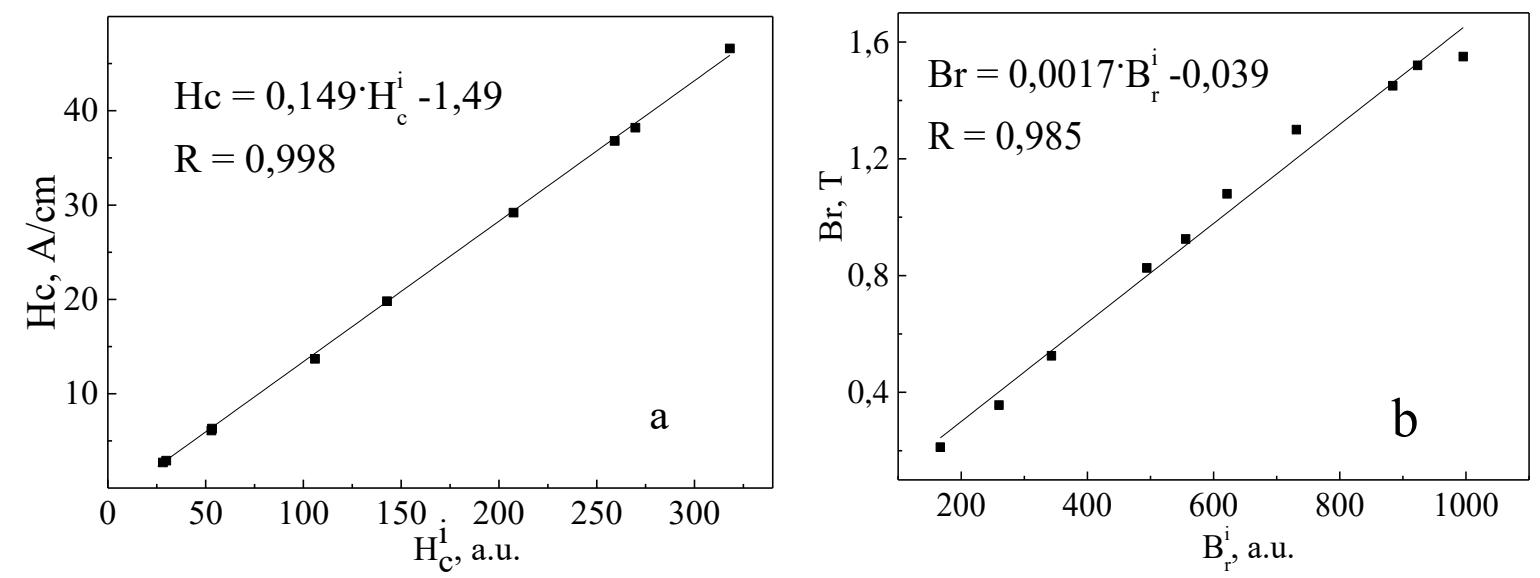

Figure 5. Equations and graphs of the calibration characteristics of DIUS-1.15M HSS by coercive force (a) and residual magnetic induction (b).
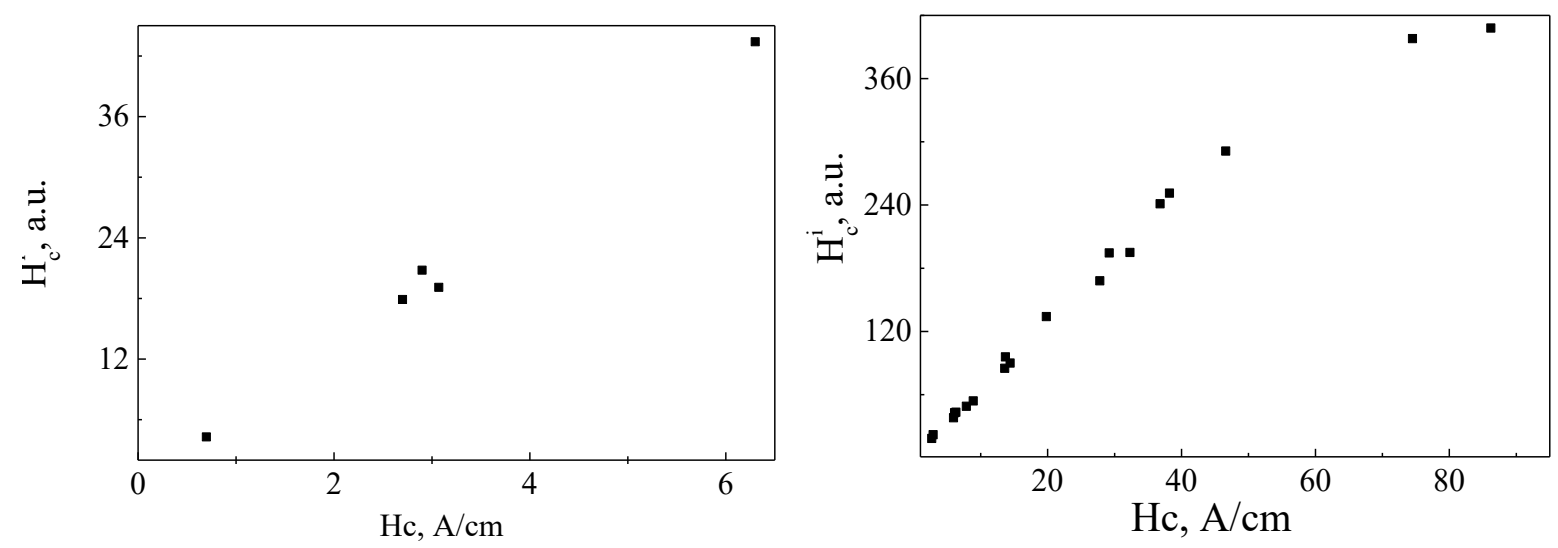

Figure 6. Calibration dependences of DIUS-1.15M HSS readings on coercive force for a range of measured small values (a) and high values of coercive force (b). 


\section{Conclusion}

As a result of the work done, the difference in the structural sensitivity of the magnetic properties locally determined in the coordinates "flow - internal field" (properties of the substance) and in the coordinates "flow in the product - current in the windings" (properties of the "body") is shown.

Also the determination of the coercive force using a conventional bipolar transducer according to the magnitude of the tangential component of the magnetic field measured near the surface of the TO in the interpolar space is shown to expand the measurement range significantly. The minimum value of the measured coercive force may even be less than the coercive force of the magnetic circuit of the electromagnet. The upper limit of measurements is determined only by the possibility of magnetization of the object being monitored, i.e. the size and shape of the transducer and the made magnetomotive force.

Thus, the developed mobile DIUS-1.15M hardware-software system could be used to solve a wide range of problems concerning with the magnetic investigation of the structure of ferromagnetic products in the presence of a correlation connection between the monitored parameters and the magnetic properties measured by the device.

\section{Acknowledgments}

The research was carried out within the state assignment of Ministry of Science and Higher Education of the Russian Federation (theme "Diagnostics" No. AAAA-A18-118020690196-3), supported in part by complex program of Ural Branch of RAS (project No. 18-10-2-8)

\section{References}

[1] Scherbinin V E and Gorkunov E S 1996 Magnetic methods of structure analysis and nondestructive testing (Ekaterinburg: UrB RAS)

[2] Filinov V V, Arakelov P G and Trubienko O V 2016 Kontrol Diagn. 5 48-52

[3] Kulak S M, Novikov V F, Probotyuk V V, Vatsenkov S M and Fursov E S 2019 Rus. J. Nondestr. Test. 55 225-32

[4] Arpan Das 2019 Materials Characterization 149 1-25

[5] Zakharov V A, Molin S M, Lenkov S V and Kolyasev V A 2018 Rus. J. Nondestr. Test. 54 5705

[6] Tiunov V F and Korzunin G S 2019 Defectoscopiya 3 46-4

[7] Yang Q, Li Y, Zhao Z, Zhu L, Luo Y and Zhu J 2014 Transactions on appl. superconduct. 24(3)

[8] Li Y, Geng X, Zhang C, Yan R and Zhu J 2017 Transactions on magnetics 53(11)

[9] Yaegashi K 2008 ISIJ International 48 461-6

[10] Sokolov R, Venedictov A, Novikov V, Kulak S 2018 Materials Today: Proceedings 11 16-25

[11] Liu K, Ma S, Ma C, Han X, Yu K, Yang S, Zhang Z, Song Y, Luo X, Chen C, Rehman S U and Zhong Z $2019 \mathrm{~J}$. of Alloys and Compunds $\mathbf{7 9 0} 78-92$

[12] Dengfeng C, Jintao Z and Haiyan X 2015 ICEMI'2015 0175 718-21

[13] Kostin V N and Smorodinskii YaG. 2017 Rus. J. Nondestr. Test. 53 493-504

[14] Kostin V N, Osintsev A A, Stashkov A N, Nichipuruk A P, Kostin K V and Sazhina E Yu 2008 Rus. J. Nondestr. Test. 44 280-9

[15] Chikazumi S 1997 Physics of Ferromagnetism(2nd edition) (Oxford University Press)

[16] Kostin V N and Vasilenko O N 2012 Rus. J. Nondestr. Test. 48 401-10

[17] Kostin V N and Vasilenko O N 2012 Rus. J. Nondestr. Test. 48 391-400 